Annuaire suisse de politique de développement

22-2 | 2003

Société de l'information et coopération internationale

\title{
Financements et partage des richesses : des sujets tabous
}

\section{Marie Thorndahl}

\section{(2) OpenEdition \\ 1 Journals}

Édition électronique

URL : http://journals.openedition.org/aspd/590

DOI : 10.4000/aspd.590

ISSN : 1663-9669

Éditeur

Institut de hautes études internationales et du développement

Édition imprimée

Date de publication : 1 novembre 2003

Pagination : 201-206

ISSN : 1660-5934

\section{Référence électronique}

Marie Thorndahl, «Financements et partage des richesses : des sujets tabous », Annuaire suisse de politique de développement [En ligne], 22-2 | 2003, mis en ligne le 23 mars 2010, consulté le 07

septembre 2020. URL : http://journals.openedition.org/aspd/590 ; DOI : https://doi.org/10.4000/aspd. 590

(c) The Graduate Institute / Geneva 


\title{
Financements et partage des richesses: des sujets tabous
}

\author{
Marie Thorndahl*
}

es sommets organisés par les Nations unies ont la réputation de créer des frustrations dues à l'écart entre les intentions et la mise en œuvre des principes et des plans d'action. Une fois qu'ils les ont adoptés, les Etats couplent rarement les textes avec des financements adéquats, ce qui crée des tensions importantes entre pays du Nord et du Sud ainsi qu'avec la société civile. Alors qu'une société de l'information équitable demande de nouvelles ressources, la réalisation du plan d'action du Sommet mondial sur la société de l'information (SMSI) s'inscrit une fois de plus dans un contexte financier particulièrement tendu.

Tout d'abord, au Nord comme au Sud, les Etats ont partout moins de capacités à mobiliser des ressources. Peu de nouveaux mécanismes de financements publics ont été inventés face à la mondialisation et à la dérégulation des économies nationales. Les taux de prélèvement sont à la baisse et de nombreuses activités économiques échappent à l'impôt (richesses liées aux réseaux électroniques et paradoxe de Solow ${ }^{1}$, paradis fiscaux, concurrences fiscales, etc.).

Les fonds alloués à la solidarité publique sont également en recul. Malgré les déclarations de bonne intention - le $0,7 \%$ du PNB consacré à l'aide publique au développement (APD) recommandés par les Nations unies -, les contributions des Etats n'ont jamais été aussi faibles: de 1990 à 1999, l'APD est passée de 0,34 à $0,24 \%$ du PNB des pays donateurs ${ }^{2}$.

Au moment de sa conception, en 1998-1999, les promoteurs du SMSI pensaient s'affranchir de cette quadrature du cercle. La folle expansion du Nasdaq laissait espérer que les sponsors privés se bousculeraient au portillon - sur le modèle

* Marie Thorndahl, socio-économiste, France.

1 Du nom de Robert Solow qui, dans les années 1950, faisait déjà le constat suivant: «Nous voyons des ordinateurs partout, sauf dans les statistiques de la productivité.» Robert Solow, «Technical Change and the Aggregate Production Function », Review of Economics and Statistics, $\mathrm{n}^{\circ}$ 39, 1957, pp. 312-320. Les économies et richesses générées par les réseaux électroniques (gains de productivité, baisse des coûts de transport, de stockage, vente de nouveaux produits, etc.) sont peu répercutées sur les prix à la consommation et sur des hausses de salaires. Elles sont surtout concentrées dans les entités privées, sans retombées sur la collectivité. Par ailleurs, le commerce électronique menace les impôts sur la consommation (type TVA), car le cyberespace ne relève d'aucun gouvernement et échappe souvent à la taxation. Les Etats commencent à se saisir du problème (notamment la Communauté européenne) mais ces questions sont loin de faire l'unanimité. Le cyberespace reste majoritairement libre de taxes.

2 PNUD, Rapport mondial sur le développement humain 2001. Mettre les nouvelles technologies au service du développement, New York; Oxford, 2001, p. 190. 
de Telecom ${ }^{3}$, la grand-messe high-tech organisée par l'Union internationale des télécommunications (UIT) - et que la manne financière s'étendrait au financement de nouveaux projets et plans d'action. Les crashes et les banqueroutes en 2000-2002 ont ruiné ce scénario et le SMSI peine même à trouver les fonds pour se financer lui-même.

Face à cette situation, quelles sont les propositions qui pourraient garantir la concrétisation d'un plan d'action et quelles sont les ouvertures politiques pour mettre en place des solutions originales au sein du SMSI?

\section{Plein d'idées, peu de recherches}

Bien qu'elles soient très diverses, la plupart des propositions de financements alternatifs peuvent être rassemblées sous le terme générique de «taxes globales » : il s'agit de lever un impôt qui sera géré au niveau international pour répondre à des problèmes globaux. Les objectifs sont les mêmes que ceux de la fiscalité nationale : changer des comportements en incitant à éviter certaines pratiques dommageables (pollutions, spéculations, etc.) et redistribuer des ressources permettant de financer des biens publics mondiaux ou des programmes de solidarité internationale.

Depuis les années 1970, les Nations unies et des mouvements citoyens ont multiplié les propositions dans ces domaines: taxes sur les transports aériens, sur la pêche internationale, sur les armes, sur les émissions de carbone, etc. Peu ont cependant fait l'objet d'études prospectives approfondies et de débats. Comme le note Luc Soete pour la bit tax, c'est un «no man's land de la recherche», parce que ces questions soulèvent des controverses partisanes qui rendent les recherches peu sereines. Le fait de s'intéresser aux taxes globales serait déjà donner de «faux signaux aux investisseurs » et mettre les économies en danger ${ }^{4}$. Ainsi, depuis les années 1990 et suite aux pressions du Congrès américain, les Nations unies ont abandonné plusieurs projets de recherche sur les taxes globales 5 .

\section{Propositions de taxes sur les réseaux électroniques}

Nous nous contenterons ici de présenter brièvement les propositions de taxes qui prennent directement appui sur les nouvelles technologies de l'information et de la communication.

<www.itu.int/WORLD2003>.

Luc Soete and Karin Kamp, The Bit Tax: The Case for Further Research, University of Maastricht, August 1996, p. 2; International Communication Round Table, Position Paper on the «Bit Tax», Brussels, July 1997.

5 Jens Martens and James A. Paul, The Coffers Are not Empty: Financing for Sustainable Development and the Role of the United Nations, July 1998, <www.globalpolicy.org/sececon/global/paul. htm>. Le niveau d'approfondissement des études est cependant très variable d'une proposition à l'autre: celles qui ont bénéficié de plus de réflexions et de débats sont les écotaxes (principe du pollueur payeur) et les taxes pour freiner les spéculations financières. 
La seule taxe inhérente aux réseaux électroniques ${ }^{6}$ qui ait largement été discutée et étudiée au niveau mondial est la taxe sur les transactions financières, mieux connue sous le nom de taxe Tobin. Le prélèvement d'un pourcentage minime sur les transactions des taux de change permettrait de réduire la spéculation et la volatilité de l'argent et de dégager d'importantes ressources pour la solidarité internationale. Taxe de correction et de redistribution donc, mais aussi taxe symbolique, qui prouverait que le système financier international peut être régulé à condition d'en avoir la volonté politique. La taxe Tobin a gagné en crédibilité technique et politique. Elle bénéficie du soutien de nombreux leaders politiques, parlementaires, syndicaux et intellectuels. Le Fonds monétaire international (FMI) et d'autres institutions, qui étaient farouchement contre elle, lui reconnaissent même quelques mérites, notamment suite à une étude élogieuse commandée par le Ministère allemand de la coopération et menée par Paul Bernd Spahn, ancien économiste du FMI ${ }^{7}$.

Une autre proposition directement issue de l'expansion d'Internet est la bit tax, qui consisterait à prélever une faible taxe indexée sur le volume du trafic Internet. Développée par le Club de Rome en 1994 et popularisée par le Programme des Nations unies pour le développement (PNUD) - qui en fait mention dans son rapport annuel $1999^{8}$-, c'est la seule taxe globale spécifiquement pensée pour lutter contre la fracture numérique. Elle a cependant suscité une virulente levée de boucliers, ce qui l'a pratiquement étouffée dans l'œuf. Suite au rapport du PNUD, le Congrès américain a notamment brandi la menace que les EtatsUnis pourraient se retirer des Nations unies si des projets de taxes globales continuaient à y être discutés 9 .

L'autre proposition portée par le PNUD - cette fois-ci dans son rapport 2001 - est l'exit tax sur la fuite des cerveaux. Elle se veut une réponse aux politiques d'importation de main-d'œuvre des pays du Nord pour soutenir leur industrie informatique ${ }^{10}$. Le PNUD estime que l'Inde perd 2 milliards de dollars par an, seulement en coûts de formation de professionnels exilés. Cette exit tax, fixée sur une base de deux mois de salaire, apporterait 1 milliard de dollars par an au pays, ce qui lui permettrait d'augmenter son budget national consacré à l'éducation supérieure d'un tiers environ ${ }^{11}$. Cette proposition n'est cependant pas conçue pour alimenter un fonds de solidarité internationale; elle serait donc peu utile dans la mise en place d'un plan d'action global.

6 L'augmentation exponentielle des transactions financières et des spéculations a été rendue possible par les réseaux électroniques. La faisabilité technique de la taxe repose sur la mise en place d'une procédure électronique dans les banques habilitées aux opérations de change.

7 Paul Bernd Spahn, On the Feasibility of a Tax on Foreign Exchange Transactions, Report commissioned by the Federal Ministry for Economic Cooperation and Development, Bonn, January 2002, $<$ http://much-magic.wiwi.uni-frankfurt.de/professoren/spahn/tobintax>.

8 UNDP, Human Development Report 1999, New York; Oxford, 1999, p. 66.

9 Prohibition on United Nations Taxation Act of 1999 (Introduced in the House), 106th Congress, 6 January 1999, <www.globalpolicy.org/socecon/glotax/biblio/uscong.htm>.

10 En 2000, les Etats-Unis ont en effet adopté de nouvelles lois pour «importer» 200'000 professionnels par an, essentiellement dans le domaine de l'informatique et des nouvelles technologies. La même politique a été adoptée par des pays européens et par le Japon. Concernant les Etats-Unis uniquement, la moitié des visas sont délivrés à des informaticiens indiens.

11 PNUD, Rapport mondial sur le développement humain 2001, op. cit., p. 92. 
D'autres idées consistent à taxer les noms de domaine, soit au niveau national (par exemple .ch), soit au niveau international (par exemple .org ou .com). Ces propositions sont cependant souvent contradictoires entre elles et très mal perçues par les pionniers et les utilisateurs d'Internet. Les arguments principaux en faveur d'une taxe sur la vente de noms de domaine reposent sur le principe de bien public global. Ressource commune, les noms de domaine demandent à être protégés des spéculateurs, tout en dégageant des ressources pour aider les secteurs non lucratifs et les pays du Sud à développer leurs réseaux ${ }^{12}$. Nouveauté depuis 2003, Internet Society, une organisation non gouvernementale, gère désormais l'extension .org et compte utiliser les plus-values pour la formation et le développement d'Internet dans les pays du Sud.

Une autre proposition est de taxer les utilisateurs des spectres des fréquences radioélectriques. L'UIT est en charge de la gestion des spectres des fréquences radioélectriques et des orbites des satellites. Ce sont des ressources naturelles finies, faisant l'objet d'une demande croissante de la part de nombreux services: fixe, mobile, radiodiffusion, recherche spatiale, météorologie, systèmes mondiaux de radiorepérage, surveillance de l'environnement, services de communication pour la surveillance des mers et des airs, etc. L'UIT, qui répartit gratuitement ces fréquences, pourrait les vendre aux gouvernements qui seraient ensuite chargés de les répartir selon leur choix. De nombreux pays taxent déjà la gestion des spectres au niveau national ${ }^{13}$; la question est de savoir si cette pratique doit être généralisée et affectée, en partie, à des projets internationaux ${ }^{14}$.

\section{Cachez ces taxes!}

Malgré plus de trente ans de discussions et controverses sur les taxes globales, les avancées sont minimes. Aucun Etat ou groupe d'Etats n'a pris l'initiative de porter le débat au sein d'instances qui pourraient avoir des compétences pour concrétiser ces projets. L'histoire récente incite aussi à la prudence.

Souvenons-nous: le Sommet du millénaire (New York, 2000), qui est une source d'inspiration permanente du SMSI, avait évacué les questions de financement tout en affichant de hautes ambitions au niveau des objectifs à atteindre. Un an plus tard à Monterrey, la Conférence des Nations unies sur le financement et le développement devait spécifiquement prendre ces problèmes à brasle-corps. Elle a en grande partie échoué et la question des taxes globales y était un sujet quasi tabou. Le comité préparatoire, qui s'est penché sur la création d'une organisation internationale de la fiscalité ${ }^{15}$, a estimé que le moment n'était pas encore venu de chercher un accord international sur une taxation des multi-

12 La règle du premier arrivé, premier servi, dessert ceux qui arrivent tard sur Internet, notamment les pays du Sud. Le développement d'IPV6, la nouvelle génération d'Internet, devrait cependant régler le problème de la rareté des noms de domaine et du contrôle des Etats-Unis sur la majorité des noms.

13 Union internationale des télécommunications, Financement de la gestion du spectre: principes, janvier 1999, disponible sur le site de l'UIT <www.itu.int>.

$14<\mathrm{http}: / /$ user.intersatx.net/jc/campaign_finance.html>.

15 Certains évoquent que l'Organisation internationale de la fiscalité pourrait aussi lever des taxes globales (taxe Tobin ou carbon tax) et œuvrer pour réduire la compétition fiscale entre pays; <www.newsmax.com/archives/articles/2002/1/2/63214.shtml>. 
nationales et sur les taxes globales. Cependant, même si les financements alternatifs n'étaient pas à l'ordre du jour, ces dernières sont mentionnées dans différents documents préparatoires, dont le rapport Zedillo ${ }^{16}$.

Résultat, le Consensus de Monterrey est très conservateur: l'aide publique au développement reste l'instrument public de financement par excellence. Le consensus prie instamment tous les pays d'accomplir des efforts concrets en direction de l'objectif de 0,7\% sans pour autant que les Etats ne prennent d'engagements fermes. Il réaffirme par ailleurs que le libre-échange et les flux d'investissements demeurent les clés de la réussite des pays pauvres, pour peu que ceux-ci se donnent les moyens d'en profiter.

La mise en place de taxes touchant les réseaux électroniques semble donc improbable, puisque les Etats ont eux-mêmes restreint leur marge de manœuvre en signant des engagements antitaxes au sein de l'Organisation mondiale du commerce (OMC). Dès 1998, 132 Etats y ont adopté une déclaration ministérielle, le Standstill Agreement for Duty-free Cyberspace. Ce «gentleman's agreement» sur la non-taxation des transmissions électroniques a été renouvelé à Doha en 2001. En outre, en février 2002, 57 membres de l'OMC - qui représentent collectivement $93 \%$ du commerce des produits liés aux technologies de l'information et de la communication - ont adopté l'Information Technology Agreement (ITA), dans lequel ils s'engagent à abolir toutes les taxes sur une longue liste de produits ${ }^{17}$. Finalement, la plupart des transactions concernant le commerce électronique seront incluses dans l'Accord général sur le commerce des services (AGCS), actuellement en négociation à l'OMC, qui vise une nouvelle fois à diminuer les taxes. Ces dispositions réduisent considérablement les options futures et les décisions qui peuvent être prises dans le cadre du SMSI.

\section{De la taxe à Netaid}

Sujet embarrassant, il a fallu attendre le $2^{\mathrm{e}}$ Comité préparatoire (Prepcom 2), en février 2003, pour que la question du financement du plan d'action du SMSI soit abordée. Le président du Sénégal, Abdoulaye Wade, a le premier appelé à une solidarité numérique par laquelle la communauté internationale s'engagerait à faire converger tous les pays à l'intérieur d'un «serpent numérique » (c'est-àdire une marge inférieure et supérieure de connectivité) pour, à terme, réduire les inégalités ${ }^{18}$. Cet effort pourrait, selon ses promoteurs sénégalais, être financé conjointement par les consommateurs, les entreprises et les Etats, en instaurant notamment un prélèvement sur les ventes de matériel informatique (par exemple, 1 dollar par ordinateur acheté ou vendu).

Le contexte du SMSI pourrait, dans certaines conditions, être assez favorable à cette proposition. Les entreprises cherchent avidement de nouveaux débouchés.

$16<$ www.un.org/esa/ffd/a55-1000.pdf>. Lors de cette conférence, il a été fortement réaffirmé que «le commerce international [est le] moteur de la croissance et du développement», Nations unies, Consensus de Monterrey, mars 2002, <www.un.org/esa/ffd/aconf198-3-f.pdf>.

17 UNCTAD, E-commerce and Development Report 2002, New York; Geneva, 2002, pp. 110-111.

18 Abdouaye Wade, intervention du 17 février 2003, Prepcom 2, Genève, <www.itu.int/wsis/docs/pc2/ visionaries/wade-fr.doc $>$. 
Les quelques dollars prélevés sur leurs ventes pourraient avoir des effets multiplicateurs et se transformer en marchés futurs, après qu'ils auront transité (et certes été ponctionnés) par le système de l'aide internationale. En ce sens, ce prélèvement sur les équipements répond au mythe du gagnant-gagnant, selon lequel les entreprises du Nord trouvent de nouveaux marchés tout en connectant les habitants insolvables du Sud. Totalement dépolitisée, cette proposition n'aura des chances d'aboutir que si elle s'éloigne de la taxe globale basée sur des prélèvements obligatoires. S'il s'agit d'alimenter la solidarité numérique par des contributions volontaires, cette proposition ressemblera à s'y méprendre à d'autres initiatives des Nations unies - telles que NetAid ${ }^{19}$ ou Global Compact $^{20}$, reposant sur des donations d'entreprises, d'Etats et d'individus - qui sont loin d'avoir prouvé leur efficacité dans la lutte contre les déséquilibres mondiaux.

Ces mécanismes de financement volontaires sont aux antipodes des revendications des mouvements citoyens qui militent pour une profonde redistribution des richesses. Selon eux, seules une taxation des transactions financières et l'annulation de la dette des pays du Sud (couplées à la lutte contre les paradis fiscaux et la concurrence fiscale) permettraient d'avancer de manière significative. Ces revendications ont peu de chance d'influencer les débats au sein du SMSI. La société civile accréditée, absorbée par des tensions internes et la recherche de financements à court terme pour pouvoir assurer sa participation aux activités du sommet, ménage ses partenaires et ne s'identifie pas forcément aux mouvements considérés comme radicaux, qui s'expriment à Porto Alegre ou Evian.

Les solutions préconisées par les participants du SMSI - notamment les partenariats publics-privés, publics-publics, les investissements directs ou encore de coopération décentralisée - offrent certainement quelques perspectives intéressantes pour financer des projets. Toutes ces propositions contournent cependant la question fondamentale du partage des richesses dans la société de l'information. L'injonction de «connecter tous les villages du monde d'ici $2010 »^{21}$ restera - tel l'objectif de la «santé pour tous d'ici l'an $2000 »^{22}$ lancé en 1979 - une autre prophétie moderne dont les sommets nous abreuvent, mais auxquelles personne ne peut plus croire sérieusement.

$19<w w w . n e t a i d . o r g>$.

$20<$ www.unglobalcompact.org $>$.

21 WSIS (World Summit on the Information Society), Draft Action Plan: Based on Discussions in the Working Group of Sub-Committee 2, document WSIS/PCIP/DT/2-E, 21 March 2003, p. 8, disponible sur le site de l'UIT <www.itu.int>.

22 La Stratégie mondiale de la santé pour tous d'ici l'an 2000 a été lancée par la 32 Assemblée mondiale de la santé en 1979, organisée par l'Organisation mondiale de la santé; <www.who.int>. 